\title{
Correction to: Effect of a Preparation of Four Probiotics on Symptoms of Patients with Irritable Bowel Syndrome: Association with Intestinal Bacterial Overgrowth
}

\author{
Konstantinos Leventogiannis ${ }^{1}$ - Paraskevas Gkolfakis ${ }^{2}$ - Georgios Spithakis ${ }^{1}$ - Aikaterini Tsatali ${ }^{1}$ - Aikaterini Pistiki ${ }^{1}$. \\ Athanasios Sioulas $^{2} \cdot$ Evangelos J. Giamarellos-Bourboulis ${ }^{1,3} \cdot$ Konstantinos Triantafyllou $^{2}$
}

Published online: 28 March 2018

(C) Springer Science+Business Media, LLC, part of Springer Nature 2018

Correction to: Probiotics and Antimicrobial Proteins (2018)

https://doi.org/10.1007/s12602-018-9401-3

The original version of this article contained mistakes, and the authors would like to correct them. The correct details are given below:

- On page 6, right column line 4, SIBO should read IBS-C.

- Figure 4, the legend of panel (d) should read IBS-M(+) $(n=10)$.

- Figure 5, the legend of panel (b) should read IBS-M(+) $(n=10)$.

The authors apologize for potential issues raised by these mistakes.

The online version of the original article can be found at https://doi.org/ 10.1007/s12602-018-9401-3

Evangelos J. Giamarellos-Bourboulis

egiamarel@med.uoa.gr

1 4th Department of Internal Medicine, National and Kapodistrian University of Athens, Athens, Greece

2 Hepatogastroenterology Unit, 2nd Department of Internal Medicine-Propedeutic, Research Institute and Diabetes Center, National and Kapodistrian University of Athens, Athens, Greece

3 4th Department of Internal Medicine, ATTIKON University Hospital, 1 Rimini Street, 1262 Athens, Greece 
a) Bowel satisfaction and SIBO

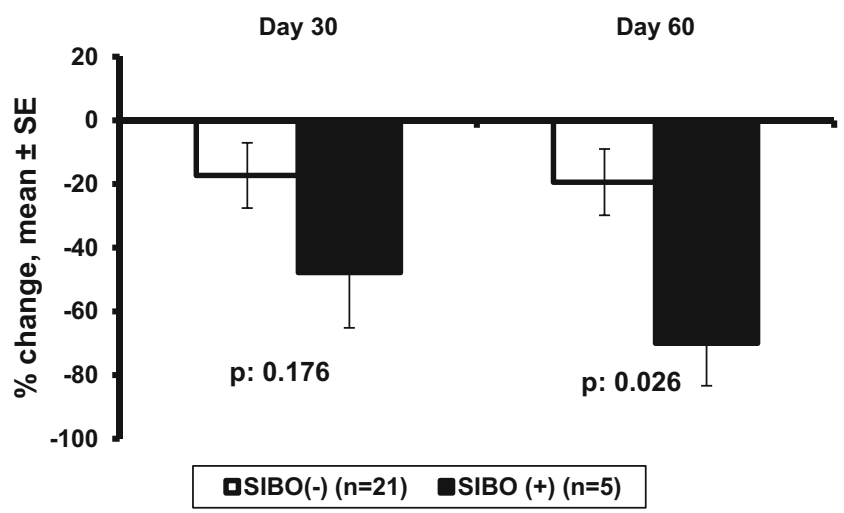

c) Bowel satisfaction and IBS-D

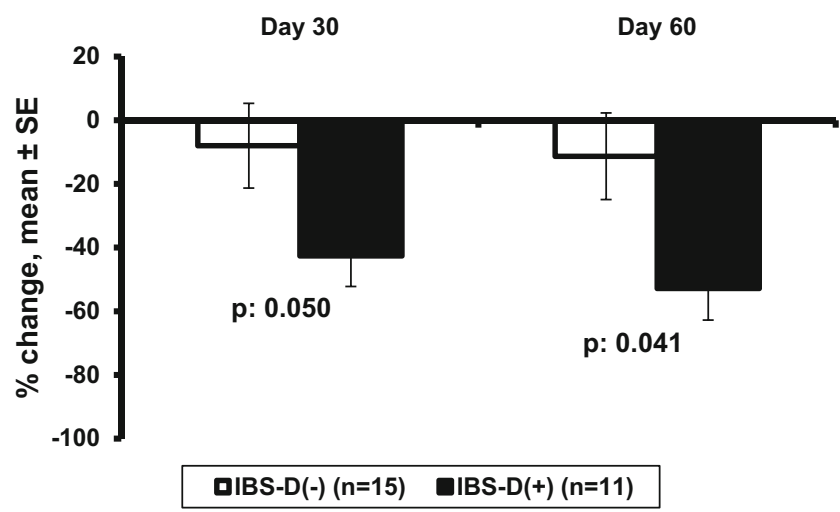

Fig. 4 Effect of treatment with the studied preparation of four probiotics on the components of the IBS Severity Scoring System. a, b Percent change of scoring for bowel satisfaction and for the effect of bowel function on daily life in relation to the absence or presence of small intestinal bacterial overgrowth (SIBO). c Percent change of scoring for b) Effect on daily life and SIBO

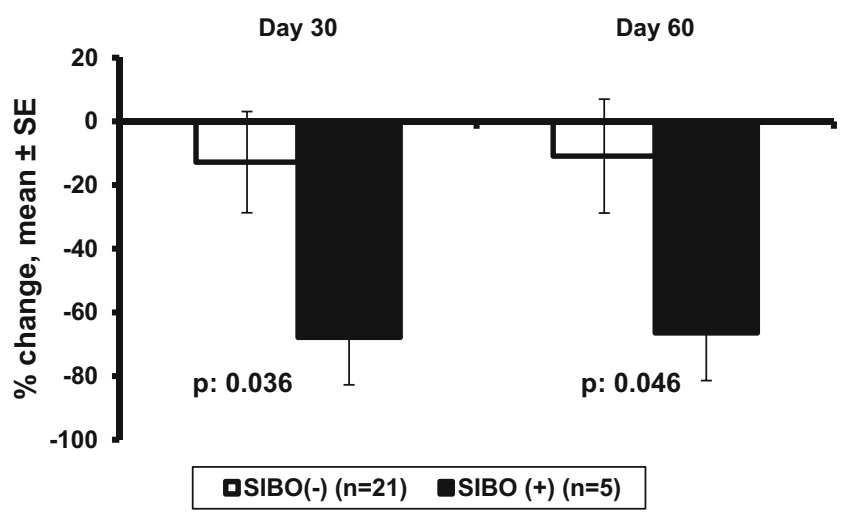

d) Pain intensity and IBS-M

Day 30 Day 60

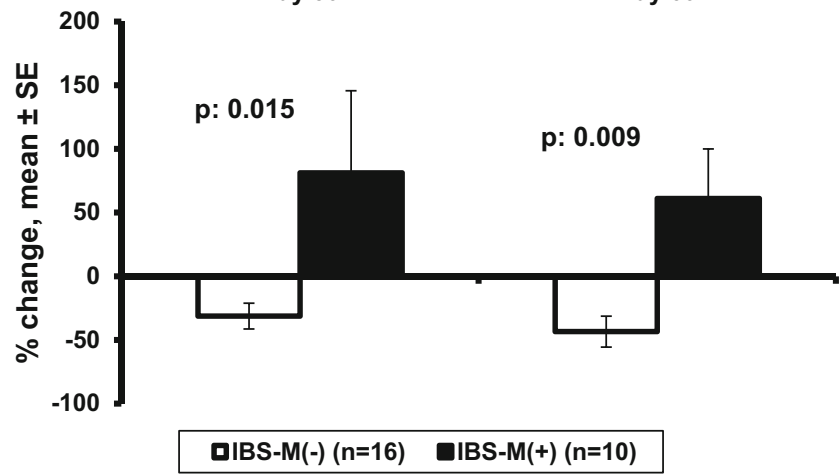

bowel function satisfaction in relation to the presence of diarrhea predominant IBS (IBD-D). d Percent change of abdominal pain intensity in relation to the presence of mixed-type IBS (IBD-M). The $p$ values of statistically significant comparisons are shown 
a) Normal Bristol stool scale and IBS-C

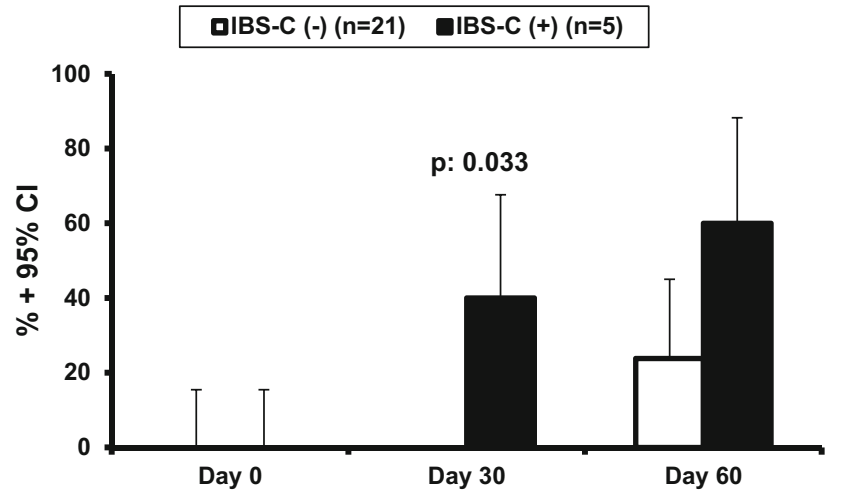

b) Change of days of work loss and IBS-M

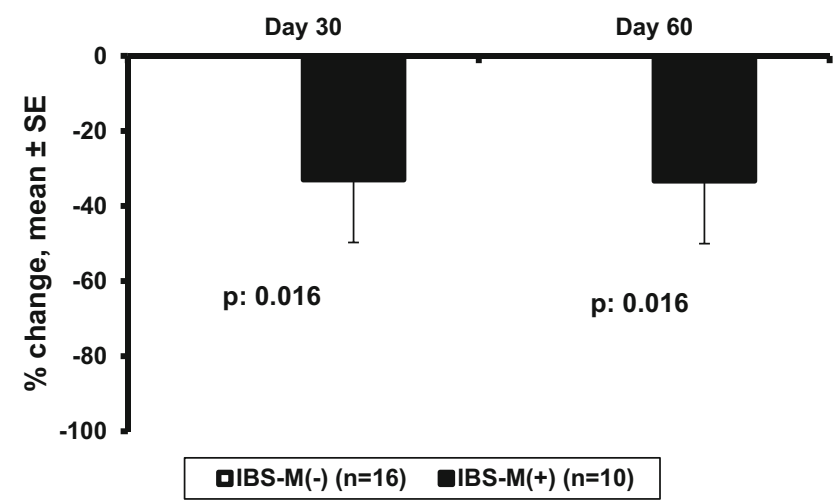

Fig. 5 Effect of treatment with the studied preparation of four probiotics on the stool form and on the days of work loss. a Rate of patients with normal stool in relation to the presence of constipation-predominant IBS (IBS-C). Normal stool is considered as any Bristol stool scale of 4 or 5. b Decrease of the days ofwork loss compared to the period before treatment in relation to the presence of mixed-type IBS (IBS-M). The $p$ values of statistically significant comparisons are shown. CI. confidence interval 